Elena Derjavina

Moscou, Russie

e_derzhavina@mail.ru

\title{
ARCHIPRÊTRE A. M. DERJAVIN ET SON ÉTUDE DU MÉNOLOGE DE ST. DIMITRI MÉTROPOLITE DE ROSTOV ${ }^{1}$
}

St Dimitri, métropolite de Rostov a écrit son Ménologe (Минеи Четии) vers la fin du XVIIe siècle. Il n’a pas été créé de travaux similaires dans l'histoire ultérieure de la littérature russe orthodoxe. Le Ménologe est devenu pour longtemps la lecture favorite du peuple russe. A. M. Derjavin a consacré presque 40 ans de sa vie à l'étude de ce grand ouvrage.

Alexandre M. Derjavin est né le 2 décembre (le 19 novembre) 1871 dans la famille d'un prêtre. Après ses études au séminaire de Iaroslavl Alexandre a été nommé surveillant de l'école de théologie (духовное училище) d'Uglitch, où il a travaillé jusqu'au 1897. L'activité religieuse et éducative du jeune prêtre a été aperçue. Nous supposons qu'en 1905-1908 il a été élu membre de la Société impériale palestinienne.

En 1909, on célébrait à Rostov le bicentenaire de la mort de Saint Dimitri de Rostov. L'organisateur de cet anniversaire était le métropolite de Rostov et de Yaroslavl Tikhon, futur patriarche. C'est apparemment à ce moment-là qu'Alexandre Mikhaïlovitch et le métropolite Tikhon ont fait leur connaissance. A cette époque le Père Alexandre a décidé d'entrer dans l'Académie théologique de Kiev. C'est ici qu'il a commencé à étudier les œuvres littéraires de St. Dimitri de Rostov, d'autant plus que la ville de Kiev était liée à la période la plus féconde de la vie de l'écrivain. Après la fin de ses études Derjavin est resté à l'Académie pour une année supplémentaire comme étudiant subventionné. Cette année-là, il a écrit son premier ouvrage scientifique sur Dimitri de Rostov. Cette thèse a ensuite formé la base du travail de toute sa vie.

(1) Le document a été écrit avec l'aide de la subvention de RGNF № 1004-00007a (directeur S. I. Kotkalo). Je voudrais remercier sincèrement Madame N. Smirnova pour son aide appartée lors de la traduction de cet article en français. 
En 1915-1917 la famille Derjavine s'est déplacée à Moscou, où Derjavin a beaucoup travaillé dans des dépôts de manuscrits. En 1917 il a terminé la première version de son étude et l'a envoyée à l'Académie théologique de Kiev. Mais à cause des désordres dus à la révolution, la thèse a été égarée par la poste. Toutefois, Alexandre n'a pas perdu son courage. Il a commencé presqu'aussitôt à reconstituer le texte d'après ses brouillons et sa mémoire. La nécessité de partir à zéro était un très bon prétexte de l'élargir et de l'approfondir. Alexandre Michailovich n'était pas pressé dans son travail, d'autant plus que les circonstances de sa vie ont repoussé l'achèvement de son étude à beaucoup plus tard.

Après la révolution le Père Alexandre servait dans l'église de la Sainte Trinité à Kapelki jusqu'à sa fermeture et destruction. La famille vivait près de l'église dans une petite maison sans étages. C'est là qu'Alexandre a été arrêté, en 1931, probablement en raison de sa relation proche avec le Patriarche Tikhon.

Le Père Alexandre a passé deux mois dans la prison Boutyrskaya. Après la condamnation (art. 58, point 10 - activité antisoviétique), il a été exilé à Oustioug, puis incarcéré dans des camps de concentration de la république Komi, à Syktyvkar, et ensuite dans le village de Haut-Votch (toutes ces localités se situaient à l'extrême nord de la Russie européenne), où il travaillait dans les camps de bûcherons. Après sa libération il a reçu la permission de choisir un lieu de résidence (à l'exception de Moscou et de Leningrad) avec son épouse. Alexandre Michailovich a choisi Rostov, une ville liée à son destin et à son étude de la vie et de l'œuvre de St Dimitri.

En février 1939, le dossier a été réexaminé et le casier judiciaire a été retiré. Les Derjavine se sont installés dans la même maison à Moscou. C'était pour Alexandre Mikhaïlovitch le temps le plus fructueux. Il n'a pas renoncé à ses recherches scientifiques, y compris à celles sur le Ménologe de Dimitri de Rostov. Maintenant il pouvait travailler dans les dépôts de manuscrits de Moscou et présenter ses résultats à la communauté scientifique de Moscou. Il a fait une présentation au département de la Russie ancienne de l'Institut de la littérature mondiale qui à ce moment-là était dirigé par N. K. Gudzij.

Mais ce n'est qu'en 1954 qu'Alexandre a mis le point dans son étude et l'a présentée au jugement spirituel et scientifique. La même année à la Laure de la Trinité-Saint-Serge, il a soutenu sa thèse - « Le Ménologe de St Dimitri, métropolite de Rostov en tant que manuscrit de l'histoire ecclésiastique et littéraire ». C'était la deuxième thèse théologique qui a été soutenue durant la période soviétique. 
L'archiprêtre Alexandre Derjavin est mort à l'âge de 92 ans. Il a été enterré au vieux cimetière de Moscou Pyatnitskoe près de l'église de l'Intercession (Pokrov) de la Vierge, où il a servi jusqu'à ses derniers jours.

La monographie de l'archiprêtre Alexandre Derjavin est pour le moment le seul ouvrage scientifique qui étudie d'une façon aussi profonde et exhaustive le Ménologe de St Dimitri. Il se compose de deux parties et de trois volumes d'annexes (chacun d'eux comprenant l'étude des vies des saints pour une période de quatre mois). La première partie de l'étude contient un bref aperçu des genres hagiographiques créés en Grèce du Ier au $X^{e}$ siècle. En outre, l'auteur examine l'hagiographie russe de la période pré-mongole et l'hagiographie du nord-est de la Russie aux XIV-XVI siècles. Il analyse d'une manière plus détaillée la situation en l'Ukraine et Biélorussie. «Les grandes œuvres de St. Dimitri ont été un outil important et nécessaire dans la lutte acharnée que le peuple orthodoxe ukrainien menait au XVII ${ }^{\mathrm{èm}}$ siècle contre le gouvernement polonais catholique en défendant sa foi et sa nationalité, » a écrit Derjavin dans son étude. Dans le deuxième chapitre de la première partie P. Alexandre parle d'une façon détaillée de la vie de St. Dimitri, des difficultés rencontrées lors de l'écriture et de l'impression de son ouvrage. Il raconte cette histoire où fusionnent les intérêts politiques, religieux et artistiques.

Dans le deuxième livre de son ouvrage Derjavin se concentre sur l'étude du Ménologe et tâche de suivre St. Dimitri pour révéler le travail minutieux et approfondi qu'il avait effectué. Comme l'a justement noté dans ses mémoires le métropolite de Volokolamsk Pitirim (Nétchaev), Derjavin «a refait l'énorme travail qui avait été fait avant lui par St. Dimitri ».

Tout d'abord, il montre l'approche critique adoptée par le Saint pour compiler le calendrier. La préparation minutieuse du calendrier lui a permis de découvrir des erreurs dans les calendriers slaves. Il passe ensuite directement à la question concernant les sources du Ménologe.

Les sources principales du travail d'Alexandre étaient les manuscrits de St. Dimitri, son Ménologe, des extraits de manuscrits reliés, des notes et les vies des saints qui n'ont pas toujours été incluses dans le texte final ; ces sources étaient rédigées en grec, latin, langues slaves orientales et polonais. Dimitri de Rostov s'adressait à la plupart des ouvrages non pas pour les copier, mais simplement pour en rendre une version complétée et vérifiée. «Au début du premier et du deuxiè- 
me livres trimestriels du Ménologe », écrivait Derjavin dans son étude, «St. Dimitri place une longue liste d'enseignants, écrivains, historiens et narrateurs qui ont composé ces livres. Au début de la plupart des vies des saints et parfois dans leur texte il a placé des indications sur la provenance de la vie toute entière ou seulement d'un épisode de celleci. Cela a incité I. A. Chliapkine à constater dans son livre «St Dimitri de Rostov et son temps » (1891) que toutes les sources avaient été indiquées par Dimitri lui-même. Derjavin a montré que cette conclusion est hâtive et erronée.

St. Dimitri lui-même se réfère à Siméon Métaphraste comme à la source de nombreuses vies des saints. Mais Derjavin a conclu que St. Dimitri connaissait mal la langue grecque. Normalement, c'est la fameuse édition des Bollandistes Acta Sanctorum ou la collection de Laurentius Surius qui se cachent sous de nombreuses références au Métaphraste ou sous les citations "от рукописцев греческих" (c.à.d., « d'après les manuscrits grecs »). D'ailleurs, les sources des bollandistes et de Surius étaient effectivement des manuscrits grecs.

Les annexes à l'ouvrage du Père Alexandre, composées de trois grands volumes, sont précieuses. Elles comprennent tout le cycle annuel. Ce sont trois volumes de textes tapés sur une machine à écrire qui représentent un examen détaillé des vies des saints pour chaque jour de l'année. A notre avis, ce sont les vies des quatre premiers mois (septembre-décembre) qui sont examinées de la façon la plus exhaustive. L'étude contient de nombreuses citations tirées non seulement de l'ouvrage de St. Dimitri, mais aussi des sources évoquées par le prélat. D'abord, c'est le Grand Ménologe du métropolite Macaire (plus loin GMM). Beaucoup de citations sont présentées en lecture parallèle, souvent provenant de trois sources différentes à la fois. On peut citer par exemple l'analyse de la Vie de St. Spyridon de Trimythonte (le 12 décembre), où des extraits sont tirés du Métaphraste, du Ménologe de Macaire et du Ménologe de St. Dimitri. Toutefois, dans la bibliographie de l'ouvrage il n'y a parfois pas d'indication claire concernant l'année et le lieu de publication de la source. Il semble que quelques uns de ces livres provenaient de la bibliothèque personnelle du chercheur, dont une grande partie a été remise à la bibliothèque de l'Académie théologique de Moscou après l'achèvement de l'ouvrage. Mais, malheureusement, sa collection n’a pas été marquée et il n'est pas possible de retrouver les livres d'A. M. Derjavin.

Derjavin avait souvent réussi à déchiffrer les indications de sources données d'une manière évasive. Voici quelques exemples: «Des 
manuscrits grecs » (le 27 janvier, la Vie de Sainte Eusébie-Xénia; le 31 janvier, Cyr et Jean ; le 7 février, Luc d'Hellas ; le 20 février, Léon, évêque de Catania), "De plusieurs auteurs » (le 3 septembre, Jean le Jeûneur ; 28 Janvier, Ephrem le Syrien ; le 9 août, Matthieu l'apôtre), "D'un martyrologe ancien » (le 29 juillet, Séraphin; le 13 décembre, Lucie la Vierge). Derjavin étudiait pourquoi Dimitri changeait les noms des personnages, leurs âges, souvent le lieu d'action. Ainsi, dans la Vie du saint Théodore Stratélates, dont la source principale est la recension métaphrastique de Surius, certains écarts du Métaphraste sont justifiés par la Vie qui se trouve dans la collection Anfologion ("Anthologie », un livre liturgique imprimé en 1619 à Kiev). Par exemple, la version latine du Métaphraste dit que le nombre de personnes qui priaient Christ avec St. Théodore était " octoginta », tandis que chez St. Dimitri, comme dans l'Anfologion, ce chiffre est 72 ; dans Métaphraste le pauvre porte «manum » (la main) d'Artémis, et dans l'Anfologion et St. Dimitri, la tête. Derjavin a divisé toutes les vies écrites par Dimitri en trois groupes en fonction du nombre et de la nature des sources utilisées.

Derjavin montre l'attitude critique du St. Dimitri envers les sources. Par exemple, Derjavin écrivait concernant certaines vies dans les Acta Sanctorum: "Parfois il y a des remarques portant sur son évaluation d'une vie. Ainsi, en annotant, dans le deuxième volume du mois de janvier, la Vie d'Athanase de Péloponnèse, le prélat ajoute ci-dessous: “Beaucoup de paroles, mais peu d'actes (дела мало)". En réécrivant toute une série de noms tirés du troisième volume de février, le saint marque en face de chaque nom : "il n'y a rien à écrire ..." ». D’après les manuscrits de Dimitri, Derjavin a examiné et extrait les Vies qui étaient présentes dans la liste, mais n'étaient pas inscrites dans le Ménologe. L'édition des preuves conformes à plusieurs sources témoigne du travail créatif et critique de Dimitri. A. M. Derjavin a étudié de nombreux exemples des altérations qu'il avait apportées, mais nous ne donnerons ici qu'un seul, la Vie de Saint Sabas (le 6 décembre). «Ses sources étaient, écrit Père Alexandre, la Vie du Saint écrite par Cyril [de Scythopolis - NdlR] et celles empruntées au GMM et à la recension métaphrastique de Surius. L'influence de l'une ou de l'autre source se fait souvent sentir. Ainsi, la patrie du Saint est "le village de Mutalaska" (весь Муталаска), de même que chez Surius (in vico Mutalasca, hoc est enim ei nomen), au lieu de 'la ville de Mutanska' (Мутанска града) dans le GMM; le frère de la mère de St Sabas s'appelle "Hermias" (chez Surius "cui nomen erat Hermias"), au lieu de Jérémie dans 
le GMM; le village d'un autre oncle de St. Sabas s'appelle Skanda ("in quodam vico nomine Scandoa" chez Surius), au lieu de Skaida dans le GMM. La rencontre de St. Sabas avec ses parents en Alexandrie, elle aussi, est décrite d'après Surius. Dans le GMM manquent les paroles du saint à ses parents (ici il y a une lacune dans le texte slavon), mais le nombre des pièces de monnaie d'or proposées par les parents à Sabas est indiqué d'après le GMM : quarante (четыредесять) au lieu de "viginti aureos" chez Surius. Le temps de la mort de St. Euthymius est indiqué d'après le GMM : "quand Anastase était patriarche à Jérusalem" tandis que chez Surius le nom du patriarche ne figure pas. En correspondance avec le GMM, St. Dimitri appelle les barbares que St. Sabas avait rencontré dans le désert "Sarrasins", tandis que Surius les appelle "Arageni", mais les figues qu'ils ont apportées portent le nom latin de "dactyle" ».

La liste des sources de la recherche d'A. M. Derjavin contient des titres de livres dans lesquels Dimitri a puisé le matériel pour ses Vies. Il n'y a que 25 points, mais elle comprend: Laurentius Surius, Vies des Saints de l'Est et l'Ouest - en latin, 2me éd. 1573-1578, en 7 volumes; Acta Sanctorum en 18 volumes pour les mois Janvier - Mai;

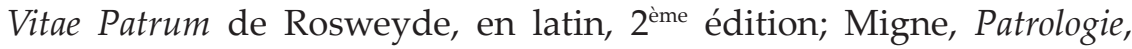
série grecque, tomes 114, 115 et 116 ; série latine, tomes 21, 73 et 74 ; Ruinart, Actes des martyrs en version originale et choisie; Baronius, Annales ecclesiastici en 12 volumes; Martyrologium Romanum, éd. 1589; historiens de l'Eglise grecque : Eusèbe Pamphile, Socrate Scholastique, Sozomène, Théodoret de Cyr, Evagre, Théodore le Lecteur - tous en version russe; Géorge Cédrène, Les lois de l'Église et de la société civile, traduction de 1794, Nicéphore Calliste, Histoire ecclésiastique, en latin, Peter Skarga, Żiwoty swętych [Vies des Saints, en polonais] ; livres de prières: calendriers, ménologes, Anfologion, Triodion etc.

Actuellement, grâce à l'Union des écrivains de la Russie, on a commencé à publier l'ouvrage d'Alexandre Michailovich Derjavin. Le premier volume est paru en 2006, et en 2008 le second.

\section{RÉSUMÉ}

Dans cet article il s'agit de l'archiprêtre Derjavin qui a réalisé l'étude du Ménologe de St. Dimitri, métropolite de Rostov. En examinant les oeuvres de cet écrivain de la fin du XVII siècle le chercheur s'arrête essentiellement sur le problème des sources liées aux Vies des Saints faisants partie du Ménologe. 